\title{
Kinetic Studies of the Reactions of Atomic Hydrogen with Iodoalkanes
}

\author{
Jessie Yuan, ${ }^{\dagger}$ Leah Wells, ${ }^{\dagger}$ and Paul Marshall $*,+, *$ \\ Department of Chemistry, University of North Texas, PO Box 5068, Denton, Texas 76203, \\ and Center for Computational Modeling of Nonstructural Materials, Wright Laboratory, \\ Wright-Patterson Air Force Base, Ohio 45433
}

Received: December 16, 1996; In Final Form: February 26, $1997^{\circledR}$

\begin{abstract}
Rate constants for the reaction of $\mathrm{H}$ atoms with the alkyl iodides iodomethane (1), deuterated iodomethane (1d), iodoethane (2), 2-iodopropane (3), and 2-iodo-2-methyl propane (4) have been measured using the flash-photolysis resonance fluorescence technique. The results are $k_{1}=(6.3 \pm 0.6) \times 10^{-11} \exp [((-5.0 \pm$ $\left.\left.0.3) \mathrm{kJ} \mathrm{mol}^{-1}\right) / R T\right](T=297-757 \mathrm{~K}), k_{1 \mathrm{~d}}=(8.0 \pm 1.7) \times 10^{-11} \exp \left[\left((-5.3 \pm 0.7) \mathrm{kJ} \mathrm{mol}^{-1}\right) / R T\right](T=$ $296-728 \mathrm{~K}), k_{2}=(1.1 \pm 0.2) \times 10^{-10} \exp \left[\left((-5.9 \pm 0.8) \mathrm{kJ} \mathrm{mol}^{-1}\right) / R T\right](T=295-624 \mathrm{~K}), k_{3}=1.4 \times 10^{-11}$ $(T=295 \mathrm{~K})$ and $k_{4}=2.0 \times 10^{-11}(T=294 \mathrm{~K}) \mathrm{cm}^{3}$ molecule $^{-1} \mathrm{~s}^{-1}$. The transition state for the substitution reaction $\mathrm{H}+\mathrm{CH}_{3} \mathrm{I} \rightarrow \mathrm{I}+\mathrm{CH}_{4}$ was characterized at the Gaussian-2 level of ab initio theory, and the process was shown to be slow. Evidence is presented showing that $\mathrm{H}$-atom abstraction is also slow, and that the dominant pathway for the reactions of $\mathrm{H}$ atoms with iodoalkanes is I-atom abstraction.
\end{abstract}

\section{Introduction}

There is growing interest in the combustion chemistry of iodine compounds, arising from the search for substitutes for the halon fire-extinguishing agents $\mathrm{CF}_{3} \mathrm{Br}$ and $\mathrm{CF}_{2} \mathrm{ClBr}^{1}$ Halon production is banned under the Montreal Protocols on Substances that Deplete the Ozone Layer. $\mathrm{CF}_{3} \mathrm{I}$ is a potential candidate for service as a new fire suppressant, but there is a lack of kinetic information on the reactions of iodine-containing compounds, especially at elevated temperatures. Modeling of the radical inhibition chemistry of $\mathrm{CF}_{3} \mathrm{I}$ suggests that significant destruction of $\mathrm{H}$-atom chain carriers occurs via the reaction

$$
\mathrm{CH}_{3} \mathrm{I}+\mathrm{H} \rightarrow \text { products }
$$

following the formation of $\mathrm{CH}_{3} \mathrm{I}$ in flames by $\mathrm{CH}_{3}+\mathrm{I}$ recombination. $^{2}$ It has been argued that reaction 1 is the dominant pathway for $\mathrm{CH}_{3} \mathrm{I}$ consumption in a stoichiometric $\mathrm{CH}_{4}$ /air flame. ${ }^{3}$

To date there have been several studies of reaction 1 at room temperature, ${ }^{4,5,6,7}$ but the temperature dependence of the rate constant $k_{1}$ has not been measured. On the basis of the thermochemistry of $\mathrm{CH}_{2} \mathrm{I}^{8}$ and other species, ${ }^{9}$ there are three exothermic product channels have been determined:

$$
\begin{aligned}
\mathrm{CH}_{3} \mathrm{I}+\mathrm{H} & \rightarrow \mathrm{CH}_{3}+\mathrm{HI} \Delta H_{298}=-61 \mathrm{~kJ} \mathrm{~mol}^{-1} \\
& \rightarrow \mathrm{CH}_{2} \mathrm{I}+\mathrm{H}_{2} \Delta H_{298}=-3 \mathrm{~kJ} \mathrm{~mol}^{-1} \\
& \rightarrow \mathrm{CH}_{4}+\mathrm{I} \Delta H_{298}=-201 \mathrm{~kJ} \mathrm{~mol}^{-1}
\end{aligned}
$$

The transition state for 1a has been characterized computationally by Schiesser et al. ${ }^{10}$ Marshall et al. ${ }^{3}$ have analyzed the transition states for channels $1 \mathrm{a}$ and $1 \mathrm{~b}$ using the Gaussian-2 methodology of Pople and co-workers, ${ }^{11}$ as extended to iodine compounds by Glukhovtsev et al., ${ }^{12}$ and derived high-temperature $\mathrm{ab}$ initio rate constants and branching ratios for $\mathrm{H}$ vs $\mathrm{I}$ abstraction. This analysis is extended in the present work to the displacement channel 1c. The temperature-dependent rate

\footnotetext{
$\dagger$ Department of Chemistry.

$¥$ Center for Computational Modeling of Nonstructural Materials.

${ }^{\otimes}$ Abstract published in Advance ACS Abstracts, April 15, 1997.
}

constant for

$$
\mathrm{CD}_{3} \mathrm{I}+\mathrm{H} \rightarrow \text { products }
$$

has also been measured, in order to assess the kinetic isotope effect.

There has been a single study ${ }^{13}$ of

$$
\mathrm{C}_{2} \mathrm{H}_{5} \mathrm{I}+\mathrm{H} \rightarrow \text { products }
$$

which yielded an experimental room temperature value of $k_{2}$ about 2 orders of magnitude smaller than literature values for $k_{1}$. The present work describes the first measurements of the temperature dependences of $k_{1}$ and $k_{2}$, and resolves the discrepancy. There is one prior determination ${ }^{13}$ of the rate constant for

$$
\mathrm{CH}_{3} \mathrm{CHICH}_{3}+\mathrm{H} \rightarrow \text { products }
$$

while

$$
\left(\mathrm{CH}_{3}\right)_{3} \mathrm{CI}+\mathrm{H} \rightarrow \text { products }
$$

appears not to have been studied previously. In this work, reactions 3 and 4 have been measured at room temperature. Structural factors that contribute to the reactivity of iodoalkanes are considered, and the reactivities of primary, secondary, and tertiary $\mathrm{C}-\mathrm{I}$ bonds are compared. Likely products are discussed, and the results are compared with ab initio information about channels $1 \mathrm{a}-\mathrm{c}$.

\section{Experimental Method}

The experimental apparatus and modifications for $\mathrm{H}$-atom kinetics have been described elsewhere. ${ }^{14,15,16}$ Briefly, atomic $\mathrm{H}$ was generated by pulsed flash lamp photolysis of $\mathrm{NH}_{3}$, through $\mathrm{MgF}_{2}$ optics, in the presence of a large excess of iodoalkane. The nominal discharge time was $7 \mu \mathrm{s}$. All experiments were carried in Ar bath gas at a total pressure $P$, and the reagent concentrations were derived from $P$ and the mole fractions of reagents in mixtures made in darkened glass bulbs. The relative concentration of $\mathrm{H}$ was monitored using time-resolved resonance fluorescence at a wavelength of 121.6 $\mathrm{nm}$. Fluorescence was detected with a solar-blind photomul- 


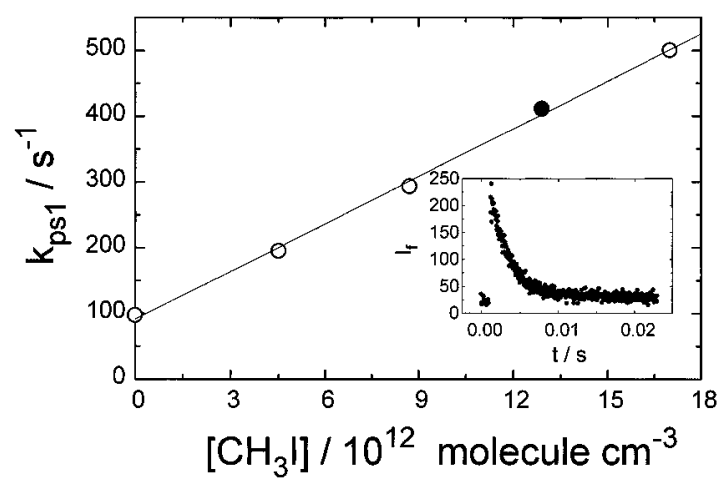

Figure 1. Plot of pseudo-first-order rate constant $k_{\mathrm{ps} 1}$ vs $\left[\mathrm{CH}_{3} \mathrm{I}\right]$ at $P$ $=70 \mathrm{mbar}$ and $T=512 \mathrm{~K}$. The inset shows the time-resolved fluorescence intensity $I_{\mathrm{f}}$ for the filled point.

tiplier tube employed with pulse counting and signal averaging. Under pseudo-first-order conditions and fixed $\left[\mathrm{NH}_{3}\right]$,

$$
\mathrm{d}[\mathrm{H}] / \mathrm{d} t=-\left(k_{X}[\mathrm{X}]+k_{\mathrm{diff}}\right)[\mathrm{H}]=-k_{\mathrm{ps} 1}[\mathrm{H}]
$$

where $\mathrm{X}$ is an iodoalkane and $k_{\mathrm{diff}}$ accounts for loss of $\mathrm{H}$ atoms out of the reaction zone other than by reaction with X. $k_{\text {diff }}$ increased as the pressure was lowered, and increased modestly as the temperature was raised, which is consistent with the idea that $k_{\text {diff }}$ reflected mainly diffusion of $\mathrm{H}$ atoms to the reactor walls. $k_{\mathrm{ps} 1}$ was obtained by fitting the observed fluorescence intensity $I_{\mathrm{f}}$ vs time profiles to an exponential decay (an example is shown as the inset on Figure 1) over typically about four to eight lifetimes. The second-order $\mathrm{H}+\mathrm{X}$ rate constant $k_{X}$ was found from linear plots of $k_{\mathrm{ps} 1}$ vs typically five values of [X], from 0 to $[\mathrm{X}]_{\max }$ (see Figure 1). This analysis is based on $[\mathrm{X}]$ $\gg[\mathrm{H}]$. Absolute $[\mathrm{H}]$ was not measured (and is not required for the determination of $k_{\mathrm{ps} 1}$, and a verification that pseudofirst-order conditions were maintained is that the derived $k_{X}$ values were independent of the initial $[\mathrm{H}]$ (see below). This quantity was altered by changing the concentration of $\mathrm{NH}_{3}$ and/ or the photolysis pulse energy. The temperature $T$ in the reaction zone was monitored with a thermocouple, corrected for radiation errors of up to $10 \mathrm{~K}$, before and after each set of $k_{X}$ measurements, and is expected to be accurate to within $\pm 2 \% .{ }^{17}$ The average residence time of gas mixtures in the heated reactor before photolysis $\tau_{\text {res }}$ was varied to check for possible pyrolysis of the iodoalkanes, while the energy discharged through the flash lamp $F$ was varied to alter the initial radical concentrations.

The Ar (Air Products, 99.997\%) was used directly from the cylinder and $\mathrm{NH}_{3}$ (MG Industries, 99.99\%) was purified by freeze-pump-thaw cycles from $77 \mathrm{~K}$. The iodoalkanes $\left(\mathrm{CH}_{3} \mathrm{I}\right.$, Aldrich, $99 \%$ and $99.5 \% ; \mathrm{CD}_{3} \mathrm{I}$, Aldrich, $99.5+$ atom \% D; $\mathrm{C}_{2} \mathrm{H}_{5} \mathrm{I}$, Lancaster, $99 \% ; \mathrm{CH}_{3} \mathrm{CHICH}_{3}$, Aldrich, $99 \%$; $\left(\mathrm{CH}_{3}\right)_{3} \mathrm{CI}$, Aldrich, 95\%) were purified by distillation to remove any iodine contamination. The $\mathrm{C}_{1}$ and $\mathrm{C}_{2}$ reagents were distilled from 273 to $77 \mathrm{~K}$, and the $\mathrm{C}_{3}$ and $\mathrm{C}_{4}$ reagents from room temperature to $77 \mathrm{~K}$.

\section{Results}

The experimental conditions and results for $k_{1}, k_{1 \mathrm{~d}}, k_{2}, k_{3}$, and $k_{4}$ are summarized in Tables $1-4$. The $k_{1}$ results were independent of the two different samples of $\mathrm{CH}_{3} \mathrm{I}$ employed. The lack of dependence of $k_{X}$ on $F$ shows that secondary chemistry involving photolysis or reaction products was negligible and that pseudo-first-order conditions were attained, and the lack of dependence of $k_{1}$ and $k_{2}$ on $\tau_{\text {res }}$ shows that pyrolysis of $\mathrm{CH}_{3} \mathrm{I}$ and $\mathrm{C}_{2} \mathrm{H}_{5} \mathrm{I}$ was unimportant at the listed temperatures.
TABLE 1: Rate Constant Measurements for $\mathbf{H}+\mathrm{CH}_{3} \mathrm{I}$

\begin{tabular}{|c|c|c|c|c|c|c|}
\hline$T, \mathrm{~K}$ & $\begin{array}{c}P, \\
\text { mbar }\end{array}$ & $\begin{array}{c}\tau_{\text {res }}, \\
\mathrm{s}\end{array}$ & $F, \mathrm{~J}$ & $\begin{array}{c}{\left[\mathrm{NH}_{3}\right], 10^{15}} \\
\text { molecule } \\
\mathrm{cm}^{-3}\end{array}$ & $\begin{array}{c}{\left[\mathrm{CH}_{3} \mathrm{I}\right]_{\max }} \\
10^{13} \text { molecule } \\
\mathrm{cm}^{-3}\end{array}$ & 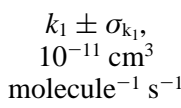 \\
\hline 295 & 82.2 & 1.7 & 4.05 & 3.35 & 8.35 & $0.83 \pm 0.02$ \\
\hline 295 & 47.5 & 1.0 & 4.05 & 1.93 & 6.08 & $0.89 \pm 0.02$ \\
\hline 295 & 47.5 & 1.0 & 6.05 & 1.93 & 4.59 & $0.86 \pm 0.01$ \\
\hline 295 & & & & & & $0.86 \pm 0.01^{a}$ \\
\hline 297 & 101.3 & 2.1 & 4.05 & 1.87 & 5.39 & $0.76 \pm 0.02$ \\
\hline 297 & 69.0 & 1.4 & 6.05 & 1.47 & 3.67 & $0.77 \pm 0.02$ \\
\hline 297 & 69.0 & 1.4 & 1.80 & 1.47 & 3.67 & $0.77 \pm 0.02$ \\
\hline 297 & 53.0 & 1.1 & 4.05 & 0.82 & 3.58 & $0.73 \pm 0.05$ \\
\hline 297 & & & & & & $0.76 \pm 0.01^{a}$ \\
\hline 362 & 130.5 & 2.2 & 4.05 & 1.19 & 4.56 & $1.29 \pm 0.03$ \\
\hline 362 & 86.7 & 1.6 & 6.05 & 1.08 & 3.99 & $1.22 \pm 0.06$ \\
\hline 362 & 86.7 & 1.6 & 1.80 & 1.08 & 3.99 & $1.21 \pm 0.07$ \\
\hline 362 & 70.1 & 0.8 & 4.05 & 0.72 & 2.73 & $1.00 \pm 0.03$ \\
\hline 362 & & & & & & $1.16 \pm 0.08^{a}$ \\
\hline 427 & 83.6 & 1.2 & 4.05 & 0.89 & 1.43 & $1.42 \pm 0.06$ \\
\hline 427 & 132.0 & 1.9 & 6.05 & 1.40 & 3.32 & $1.60 \pm 0.03$ \\
\hline 427 & 132.0 & 1.9 & 1.80 & 1.40 & 3.32 & $1.59 \pm 0.04$ \\
\hline 427 & 68.5 & 0.7 & 4.05 & 0.62 & 1.54 & $1.38 \pm 0.02$ \\
\hline 427 & & & & & & $1.45 \pm 0.08^{a}$ \\
\hline 512 & 70.7 & 1.2 & 4.05 & 1.28 & 2.42 & $2.06 \pm 0.11$ \\
\hline 512 & 110.6 & 1.9 & 4.05 & 1.73 & 2.57 & $1.95 \pm 0.05$ \\
\hline 512 & 52.1 & 0.6 & 4.05 & 0.82 & 1.59 & $1.85 \pm 0.11$ \\
\hline 512 & 50.5 & 0.9 & 4.05 & 0.99 & 2.43 & $1.97 \pm 0.08$ \\
\hline 512 & & & & & & $1.96 \pm 0.03^{a}$ \\
\hline 621 & 87.2 & 1.0 & 4.05 & 1.13 & 1.50 & $2.13 \pm 0.09$ \\
\hline 621 & 160.8 & 1.9 & 4.05 & 1.41 & 1.70 & $2.34 \pm 0.05$ \\
\hline 621 & 59.4 & 0.6 & 4.05 & 0.67 & 1.07 & $2.21 \pm 0.10$ \\
\hline 621 & 108.6 & 1.8 & 4.05 & 1.13 & 2.05 & $2.27 \pm 0.09$ \\
\hline 621 & & & & & & $2.28 \pm 0.05^{a}$ \\
\hline 757 & 111.1 & 1.3 & 4.05 & 1.26 & 1.66 & $3.00 \pm 0.07$ \\
\hline 757 & 60.0 & 0.5 & 4.05 & 0.71 & 0.88 & $2.61 \pm 0.15$ \\
\hline 757 & 164.3 & 1.3 & 4.05 & 1.47 & 1.66 & $2.86 \pm 0.08$ \\
\hline 757 & 75.6 & 0.9 & 4.05 & 0.83 & 1.13 & $2.63 \pm 0.20$ \\
\hline 757 & & & & & & $2.89 \pm 0.08^{a}$ \\
\hline
\end{tabular}

${ }^{a}$ Average value.

TABLE 2: Rate Constant Measurements for $\mathbf{H}+\mathbf{C D}_{\mathbf{3}} \mathrm{I}$

\begin{tabular}{|c|c|c|c|c|c|c|}
\hline$T, \mathrm{~K}$ & $F, \mathrm{~J}$ & $\tau, \mathrm{s}$ & $\begin{array}{c}P, \\
\text { mbar }\end{array}$ & $\begin{array}{c}{\left[\mathrm{NH}_{3}\right],} \\
10^{15} \mathrm{~cm}^{-3} \\
\text { molecule }\end{array}$ & $\begin{array}{l}{\left[\mathrm{CD}_{3} \mathrm{I}\right]_{\max },} \\
10^{12} \mathrm{~cm}^{-3} \\
\text { molecule }\end{array}$ & $\begin{array}{c}k_{1 \mathrm{~d}} \pm \sigma_{k_{1 \mathrm{~d}}} \\
10^{11} \mathrm{~cm}^{3} \\
\text { molecule }{ }^{-1} \mathrm{~s}^{-1}\end{array}$ \\
\hline 296 & 4.05 & 1.8 & 89.8 & 4.53 & 53.3 & $1.00 \pm 0.04$ \\
\hline 296 & 6.05 & 3.5 & 117.2 & 5.78 & 54.1 & $0.95 \pm 0.07$ \\
\hline 296 & 2.45 & 3.5 & 117.2 & 5.78 & 54.1 & $0.91 \pm 0.08$ \\
\hline 296 & 4.05 & 1.3 & 62.4 & 2.77 & 25.4 & $\begin{array}{l}1.09 \pm 0.06 \\
1.00 \pm 0.03^{a}\end{array}$ \\
\hline 391 & 4.05 & 1.4 & 87.6 & 1.24 & 19.9 & $1.34 \pm 0.05$ \\
\hline 391 & 6.05 & 0.9 & 58.7 & 1.34 & 15.6 & $1.43 \pm 0.03$ \\
\hline 391 & 2.45 & 0.9 & 58.7 & 1.34 & 15.6 & $1.36 \pm 0.06$ \\
\hline 391 & 4.05 & 0.7 & 85.8 & 0.98 & 11.6 & $\begin{array}{l}1.48 \pm 0.04 \\
1.42 \pm 0.03^{a}\end{array}$ \\
\hline 516 & 4.05 & 1.0 & 84.9 & 1.47 & 8.8 & $2.33 \pm 0.21$ \\
\hline 516 & 4.05 & 0.5 & 74.6 & 0.95 & 7.7 & $2.35 \pm 0.13$ \\
\hline 516 & 4.05 & 1.6 & 136.6 & 1.96 & 9.8 & $\begin{array}{l}2.58 \pm 0.08 \\
2.50 \pm 0.08^{a}\end{array}$ \\
\hline 728 & 4.05 & 0.8 & 90.7 & 0.60 & 6.63 & $3.62 \pm 0.14$ \\
\hline 728 & 4.05 & 0.4 & 91.0 & 0.35 & 6.15 & $3.06 \pm 0.24$ \\
\hline 728 & 4.05 & 0.3 & 66.9 & 0.27 & 5.82 & $3.41 \pm 0.20$ \\
\hline 728 & 4.05 & 0.9 & 70.8 & 0.69 & 7.74 & $\begin{array}{l}3.30 \pm 0.13 \\
3.40 \pm 0.11^{a}\end{array}$ \\
\hline
\end{tabular}

${ }^{a}$ Average value.

Data for reactions 1 and 2 at higher temperatures did show consistent variation with $\tau_{\text {res }}$, and for reaction 2 showed a decrease in the apparent $k_{2}$ above $630 \mathrm{~K}$, and therefore were excluded from further analysis.

The Arrhenius plot for reaction 1 is shown in Figure 2, and the weighted linear fit yields

$$
\begin{array}{r}
k_{1}=(6.3 \pm 0.6) \times 10^{-11} \exp \left[\left((-5.0 \pm 0.3) \mathrm{kJ} \mathrm{mol}^{-1}\right) /\right. \\
R T] \mathrm{cm}^{3} \text { molecule }^{-1} \mathrm{~s}^{-1}
\end{array}
$$

The quoted errors in the Arrhenius parameters are $1 \sigma$ and are 
TABLE 3: Rate Constant Measurements for $\mathbf{H}+\mathbf{C}_{2} \mathbf{H}_{5} \mathbf{I}$

\begin{tabular}{|c|c|c|c|c|c|c|}
\hline$T, \mathrm{~K}$ & $\begin{array}{c}P, \\
\text { mbar }\end{array}$ & $\begin{array}{c}\tau_{\text {res }}, \\
\mathrm{s}\end{array}$ & $F, \mathrm{~J}$ & $\begin{array}{c}{\left[\begin{array}{c}\left.\mathrm{NH}_{3}\right] \\
10^{15} \mathrm{molecule} \\
\mathrm{cm}^{-3}\end{array}\right.}\end{array}$ & $\begin{array}{c}{\left[\mathrm{C}_{2} \mathrm{H}_{5} \mathrm{I}\right]_{\max }} \\
10^{13} \text { molecule } \\
\mathrm{cm}^{-3}\end{array}$ & $\begin{array}{c}k_{2} \pm \sigma_{k_{2}} \\
10^{-11} \mathrm{~cm}^{3} \\
\text { molecule }{ }^{-1} \mathrm{~s}^{-}\end{array}$ \\
\hline 295 & 76.5 & 1.6 & 4.05 & 1.22 & 4.62 & $1.00 \pm 0.03$ \\
\hline 295 & 55.5 & 0.9 & 6.05 & 0.94 & 4.18 & $0.92 \pm 0.02$ \\
\hline 295 & 55.5 & 0.9 & 1.80 & 0.94 & 4.18 & $0.85 \pm 0.03$ \\
\hline 295 & 109.5 & 1.7 & 4.05 & 0.66 & 6.62 & $1.06 \pm 0.04$ \\
\hline 295 & & & & & & $0.94 \pm 0.04^{a}$ \\
\hline 357 & 131.8 & 2.3 & 4.05 & 1.23 & 3.92 & $1.42 \pm 0.04$ \\
\hline 357 & 88.4 & 1.5 & 6.05 & 1.20 & 5.08 & $1.59 \pm 0.02$ \\
\hline 357 & 88.4 & 1.5 & 1.80 & 1.20 & 5.08 & $1.50 \pm 0.03$ \\
\hline 357 & 67.4 & 0.8 & 4.05 & 0.65 & 3.48 & $1.36 \pm 0.05$ \\
\hline 357 & & & & & & $1.53 \pm 0.05^{a}$ \\
\hline 450 & 132.8 & 1.8 & 4.05 & 0.86 & 3.05 & $1.95 \pm 0.05$ \\
\hline 450 & 91.3 & 1.2 & 6.05 & 1.49 & 4.15 & $2.29 \pm 0.03$ \\
\hline 450 & 91.3 & 1.2 & 1.80 & 1.49 & 4.15 & $2.22 \pm 0.03$ \\
\hline 450 & 68.0 & 0.7 & 4.05 & 0.83 & 2.89 & $2.21 \pm 0.08$ \\
\hline 450 & & & & & & $2.21 \pm 0.06^{a}$ \\
\hline 533 & 129.5 & 1.8 & 4.05 & 0.99 & 2.71 & $2.61 \pm 0.03$ \\
\hline 533 & 85.0 & 1.0 & 6.05 & 0.55 & 1.99 & $2.65 \pm 0.05$ \\
\hline 533 & 85.0 & 1.0 & 1.80 & 0.55 & 1.99 & $2.55 \pm 0.07$ \\
\hline 533 & 69.4 & 0.6 & 4.05 & 0.33 & 1.79 & $2.36 \pm 0.08$ \\
\hline 533 & & & & & & $2.59 \pm 0.04^{a}$ \\
\hline 624 & 155.6 & 2.0 & 4.05 & 1.10 & 3.02 & $3.95 \pm 0.07$ \\
\hline 624 & 96.9 & 0.9 & 4.05 & 0.49 & 1.79 & $3.75 \pm 0.13$ \\
\hline 624 & 153.8 & 1.8 & 4.05 & 0.70 & 2.75 & $3.88 \pm 0.16$ \\
\hline 624 & 76.6 & 0.6 & 4.05 & 0.35 & 1.30 & $3.58 \pm 0.09$ \\
\hline 624 & & & & & & $3.80 \pm 0.09^{a}$ \\
\hline
\end{tabular}

${ }^{a}$ Average value.

statistical only. Consideration of the covariance leads to a $1 \sigma$ precision for the fitted $k_{1}$ of $3-5 \%$, and allowance for possible systematic errors leads to $95 \%$ confidence intervals of $\pm 13 \%$. The Arrhenius plot for reaction 1d is also shown in Figure 2, and the weighted linear fit yields

$$
\begin{array}{r}
k_{1 \mathrm{~d}}=(8.0 \pm 1.7) \times 10^{-11} \exp \left[\left((-5.3 \pm 0.7) \mathrm{kJ} \mathrm{mol}^{-1}\right) /\right. \\
R T] \mathrm{cm}^{3} \text { molecule } \mathrm{s}^{-1}
\end{array}
$$

Consideration of the covariance leads to a $1 \sigma$ precision for the fitted $k_{1 \mathrm{~d}}$ of $6-10 \%$, and allowance for possible systematic errors leads to $95 \%$ confidence intervals of $\pm 19 \%$.

The Arrhenius plot for reaction 2 is also shown on Figure 2, and the best fit is

$$
\begin{array}{r}
k_{2}=(1.1 \pm 0.2) \times 10^{-10} \exp \left[\left((-5.9 \pm 0.8) \mathrm{kJ} \mathrm{mol}^{-1}\right) /\right. \\
R T] \mathrm{cm}^{3} \text { molecule } \mathrm{s}^{-1}
\end{array}
$$

The $1 \sigma$ precision of the fitted $k_{2}$ is $5-13 \%$, and allowance for possible systematic errors leads to $95 \%$ confidence intervals of $\pm 28 \%$ at the extremes of the experimental $T$ range to $\pm 14 \%$ at the center. Similar accuracies, about $\pm 20 \%$, are expected for reactions 3 and 4 which were studied at room temperature only (see Table 4).

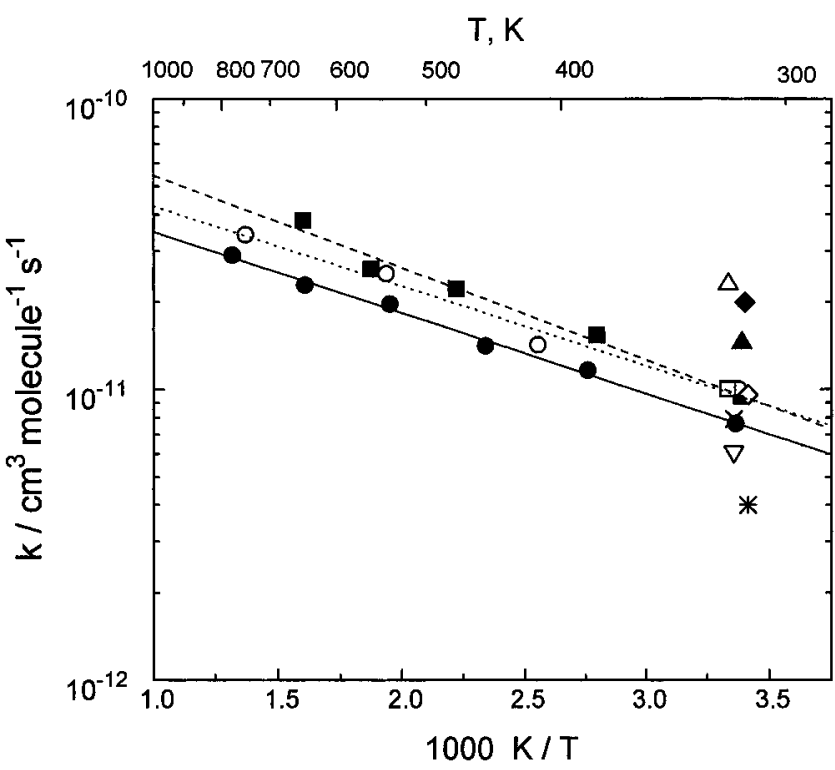

Figure 2. Arrhenius plot of rate constants for $\mathrm{H}$ reactions with $\mathrm{CH}_{3} \mathrm{I}$ $\left(\bullet\right.$ and solid line, this work; $X$ ref $4 ; \diamond$, ref $5 ; \nabla$, ref $6 ; *$, ref 7 ), $\mathrm{CD}_{3} \mathrm{I}$ ( $\mathrm{O}$ and dotted line, this work), $\mathrm{C}_{2} \mathrm{H}_{5} \mathrm{I}$ ( $\square$ and dashed line, this work; $\square$, ref 13 corrected), $\mathrm{C}_{3} \mathrm{H}_{7} \mathrm{I}$ ( $\Delta$, this work; $\Delta$, ref 13 corrected) and $\mathrm{C}_{4} \mathrm{H}_{9} \mathrm{I}$ $(\diamond$, this work).

\section{Discussion}

Our $k_{1}$ values are compared with four previous measurements in Figure 2 and Table 5, and it may be seen that there is particularly good accord with the most recent literature value, which was obtained by a similar technique. ${ }^{7}$ There is a single previous determination of $k_{2}$, based on a rate measurement relative to

$$
\mathrm{H}+\mathrm{HI} \rightarrow \mathrm{H}_{2}+\mathrm{I}
$$

and which Rebbert et al. reported as $k_{2}=7 \times 10^{-14} \mathrm{~cm}^{3}$ molecule $\mathrm{e}^{-1} \mathrm{~s}^{-1} \cdot{ }^{13}$ This is a factor of 150 smaller than measured here, because Rebbert et al. apparently used an incorrect value of $k_{9}=1.7 \times 10^{-13} \mathrm{~cm}^{3}$ molecule ${ }^{-1} \mathrm{~s}^{-1} .^{13}$ At room temperature $k_{9} \approx 2.4 \times 10^{-11} \mathrm{~cm}^{3}$ molecule ${ }^{-1} \mathrm{~s}^{-1} \cdot{ }^{18,19,20}$ Using the same $k_{2} / k_{9}$ as Rebbert et al., the corrected $k_{2}$ is then $1.0 \times 10^{-11} \mathrm{~cm}^{3}$ molecule ${ }^{-1} \mathrm{~s}^{-1}$, close to the present value. The same explanation accounts for much of the discrepancy between the value of $k_{3}$ given by Rebbert et al., ${ }^{13} 1.6 \times 10^{-13} \mathrm{~cm}^{3}$ molecule ${ }^{-1}$ $\mathrm{s}^{-1}$, and our own direct measurement (Table 5) which is 90 times higher. The corrected $k_{3}$ is $2.3 \times 10^{-11} \mathrm{~cm}^{3}$ molecule ${ }^{-1}$ $\mathrm{s}^{-1}$ which is in good accord with our result. There appear to be no literature values for $k_{4}$.

As noted in the introduction, three possible reaction pathways

\begin{tabular}{|c|c|c|c|c|c|c|c|}
\hline iodoalkane, X & $T, \mathrm{~K}$ & $P$, mbar & $\tau_{\text {res }}, \mathrm{s}$ & $F, \mathrm{~J}$ & {$\left[\mathrm{NH}_{3}\right], 10^{15}$ molecule $\mathrm{cm}^{-3}$} & {$[\mathrm{X}]_{\max }, 10^{13}$ molecule $\mathrm{cm}^{-3}$} & $k_{X} \pm \sigma_{k_{X}}, 10^{-11} \mathrm{~cm}^{3}$ molecule ${ }^{-1} \mathrm{~s}^{-1}$ \\
\hline \multirow[t]{5}{*}{$\mathrm{CH}_{3} \mathrm{CHICH}_{3}$} & 295 & 129.0 & 2.0 & 4.05 & 1.19 & 4.87 & $1.49 \pm 0.02$ \\
\hline & 295 & 77.5 & 1.2 & 1.80 & 0.71 & 3.64 & $1.39 \pm 0.02$ \\
\hline & 295 & 77.5 & 1.2 & 6.05 & 0.71 & 3.64 & $1.42 \pm 0.03$ \\
\hline & 295 & 66.0 & 0.8 & 4.05 & 0.44 & 2.70 & $1.48 \pm 0.04$ \\
\hline & 295 & & & & & & $1.44 \pm 0.03^{a}$ \\
\hline \multirow[t]{5}{*}{$\left(\mathrm{CH}_{3}\right)_{3} \mathrm{CI}$} & 294 & 129.6 & 2.7 & 4.05 & 1.47 & 4.34 & $1.88 \pm 0.05$ \\
\hline & 294 & 84.3 & 1.3 & 6.05 & 1.04 & 3.11 & $2.05 \pm 0.04$ \\
\hline & 294 & 84.3 & 1.3 & 1.80 & 1.04 & 3.11 & $2.00 \pm 0.04$ \\
\hline & 294 & 68.9 & 0.7 & 4.05 & 0.53 & 2.10 & $1.95 \pm 0.07$ \\
\hline & 294 & & & & & & $1.99 \pm 0.04^{a}$ \\
\hline
\end{tabular}
for the $\mathrm{H}$ plus iodoalkane reactions are I-abstraction, $\mathrm{H}$ abstraction, and I-substitution. These are all bimolecular processes, consistent with the observed pressure independence

TABLE 4: Rate Constant Measurements for $\mathrm{H}+\mathrm{CH}_{3} \mathrm{CHICH}_{3}$ and $\left(\mathrm{CH}_{3}\right)_{3} \mathrm{CI}$

${ }^{a}$ Average value. 
TABLE 5: Summary of $\mathbf{H}+$ Iodoalkane Measurements

\begin{tabular}{|c|c|c|c|c|c|}
\hline reaction & method & 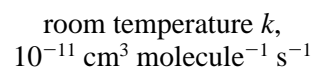 & $\begin{array}{l}A, 10^{-11} \mathrm{~cm}^{3} \\
\text { molecule }{ }^{-1} \mathrm{~s}^{-1}\end{array}$ & $E_{\mathrm{a}}, \mathrm{kJ} \mathrm{mol}^{-1}$ & lit. \\
\hline \multirow{5}{*}{$\mathrm{H}+\mathrm{CH}_{3} \mathrm{I}$} & discharge flow-mass spectrometry & $0.4 \pm 0.1$ & & & ref 4 \\
\hline & flash photolysis-UV absorption & $0.96 \pm 0.05$ & & & ref 5 \\
\hline & pulsed radiolysis - IR absorption & $0.61 \pm 0.05$ & & & ref 6 \\
\hline & flash photolysis-resonance fluorescence & $0.79 \pm 0.08$ & & & ref 7 \\
\hline & flash photolysis -resonance fluorescence & $0.83 \pm 0.11$ & $6.3 \pm 0.6$ & $5.0 \pm 0.3$ & this work \\
\hline $\mathrm{H}+\mathrm{CD}_{3} \mathrm{I}$ & flash photolysis-resonance fluorescence & $0.94 \pm 0.18$ & $8.0 \pm 1.7$ & $5.3 \pm 0.7$ & this work \\
\hline \multirow[t]{2}{*}{$\mathrm{H}+\mathrm{C}_{2} \mathrm{H}_{5} \mathrm{I}$} & steady photolysis - relative to $\mathrm{H}+\mathrm{HI}$ & $1^{a}\left(0.007^{b}\right)$ & & & ref 13 \\
\hline & flash photolysis -resonance fluorescence & $1.0 \pm 0.3$ & $1.1 \pm 0.2$ & $5.9 \pm 0.8$ & this work \\
\hline \multirow{2}{*}{$\mathrm{H}+\mathrm{CH}_{3} \mathrm{CHICH}_{3}$} & steady photolysis - relative to $\mathrm{H}+\mathrm{HI}$ & $2.3^{a}\left(0.016^{b}\right)$ & & & ref 13 \\
\hline & flash photolysis - resonance fluorescence & $1.4 \pm 0.3$ & & & this work \\
\hline $\mathrm{H}+\left(\mathrm{CH}_{3}\right)_{3} \mathrm{CI}$ & flash photolysis - resonance fluorescence & $2.0 \pm 0.4$ & & & this work \\
\hline
\end{tabular}

${ }^{a}$ Corrected (see text). ${ }^{b}$ Uncorrected literature value.

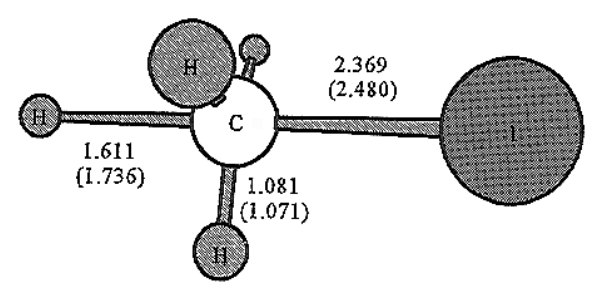

Figure 3. $\mathrm{Ab}$ initio geometries of the $C_{3 v}$ transition state for $\mathrm{H}+$ $\mathrm{CH}_{3} \mathrm{I} \rightarrow \mathrm{CH}_{4}+\mathrm{I}$. MP2=full/6-31G(d) data shown $(\mathrm{HF} / 6-31 \mathrm{G}(\mathrm{d})$ in parentheses). Distances are in $10^{-10} \mathrm{~m}$ and the ICH angles are $96.6^{\circ}$ $\left(95.8^{\circ}\right)$.

TABLE 6: Ab Initio Results for the Transition State for $\mathbf{H}$ $+\mathrm{CH}_{3} \mathrm{I} \rightarrow \mathrm{CH}_{4}+\mathrm{I}$

\begin{tabular}{|c|c|}
\hline $\mathrm{HF} / 6-31 \mathrm{G}(\mathrm{d})$ frequencies ${ }^{a}$ & $\begin{array}{l}1199 \mathrm{i}, 348,410(2), 1082(2), \\
\quad 1187,1541(2), 3313,3495(2)\end{array}$ \\
\hline $\mathrm{MP} 2=$ full/6-31G $(\mathrm{d})$ frequencies ${ }^{a}$ & $\begin{array}{l}1674 \mathrm{i}, 463,470(2), 1160(2), \\
\quad 1255,1464(2), 3193,3375(2)\end{array}$ \\
\hline MP2/6-311G(d,p $)^{b}$ & -6957.17377 \\
\hline MP4/6-311G(d,p $)^{b}$ & -6957.21271 \\
\hline $\mathrm{QCISD}(\mathrm{T}) / 6-311 \mathrm{G}(\mathrm{d}, \mathrm{p})^{b}$ & -6957.21898 \\
\hline $\mathrm{MP} 2 / 6-311+\mathrm{G}(\mathrm{d}, \mathrm{p})^{b}$ & -6957.17498 \\
\hline $\mathrm{MP} 4 / 6-311+\mathrm{G}(\mathrm{d}, \mathrm{p})^{b}$ & -6957.21407 \\
\hline $\mathrm{MP} 2 / 6-311 \mathrm{G}(2 \mathrm{df}, \mathrm{p})^{b}$ & -6957.23020 \\
\hline $\mathrm{MP} 4 / 6-311 \mathrm{G}(2 \mathrm{df}, \mathrm{p})^{b}$ & -6957.27893 \\
\hline $\mathrm{MP} 2 / 6-311+\mathrm{G}(3 \mathrm{df}, 2 \mathrm{p})^{b}$ & -6957.25902 \\
\hline G2[all-electron] ${ }^{b}$ & -6957.31294 \\
\hline
\end{tabular}

${ }^{a}$ Unscaled, in $\mathrm{cm}^{-1}$. (2) denotes doubly degenerate modes. ${ }^{b} \mathrm{G} 2$ energy component, in au; $1 \mathrm{au} \approx 2625 \mathrm{~kJ} \mathrm{~mol}^{-1}$.

of the rate constants. Searches at up to the QCISD/6-311G$(d, p)$ level of theory showed no evidence of a bound $\mathrm{H}+\mathrm{CH}_{3} \mathrm{I}$ intermediate, whose formation would likely to be pressure dependent. The most exothermic of the bimolecular channels is displacement of the I atom. We have characterized the transition state for this process at the HF/6-31G(d) and MP2=full/6-31G(d) levels of theory, and the geometry is shown in Figure 3. Higher level single-point energy calculations yielded the G2 energy (see Table 6) which approximates a QCISD(T)/6-311+G(3df,2p) result. ${ }^{11,12}$ Computations were carried out with the Gaussian 94 program suite. ${ }^{21}$ Relative to the $\mathrm{G} 2$ energy of $\mathrm{H}+\mathrm{CH}_{3} \mathrm{I},{ }^{12}$ the $\mathrm{G} 2$ barrier to substitution at $0 \mathrm{~K}$ is predicted to be $45 \mathrm{~kJ} \mathrm{~mol}^{-1}$. The size of this barrier indicates that I-atom displacement is kinetically unfavorable. Furthermore, the transition state for this process is found to be tight. The unscaled MP2 =full/6-31G(d) frequencies (Table 6) and the geometry, together with entropy data for $\mathrm{H}$ and $\mathrm{CH}_{3} \mathrm{I},{ }^{22}$ lead to an entropy of activation for reaction 1c of $\Delta S^{\ddagger}{ }_{298}=$ $-91 \mathrm{~J} \mathrm{~K}^{-1} \mathrm{~mol}^{-1}$ and an implied preexponential factor at 298 $\mathrm{K}$ of about $2 \times 10^{-13} \mathrm{~cm}^{3}$ molecule $\mathrm{e}^{-1} \mathrm{~s}^{-1}$, more than 2 orders of magnitude below that observed. Substitution will therefore make a negligible contribution to the total $k_{1}$.
TABLE 7: Comparison of Iodoalkane Properties at Room Temperature

\begin{tabular}{lccc}
\hline $\begin{array}{c}\text { iodoalkane, } \\
\mathrm{X}\end{array}$ & $\begin{array}{c}k(298 \mathrm{~K}) \text { for } \\
\mathrm{H} \text { abstraction, } \\
\mathrm{cm}^{3} \text { molecule }\end{array} \mathrm{s}^{-1}$ & $\begin{array}{c}\text { measured } k_{X} \\
(298 \mathrm{~K}), \mathrm{cm}^{3} \\
\text { molecule }^{-1} \mathrm{~s}^{-1}\end{array}$ & $\begin{array}{c}\mathrm{DH}_{298}(\mathrm{C}-\mathrm{I}),{ }^{b} \\
\mathrm{~kJ} \mathrm{~mol}^{-1}\end{array}$ \\
\hline $\mathrm{CH}_{3} \mathrm{I}$ & $5.6 \times 10^{-19}$ & $8.3 \times 10^{-12}$ & $237.2 \pm 1.3$ \\
$\mathrm{C}_{2} \mathrm{H}_{5} \mathrm{I}$ & $3.8 \times 10^{-17}$ & $1.0 \times 10^{-11}$ & $236.8 \pm 1.7$ \\
$\mathrm{CH}_{3} \mathrm{CHICH}$ & $5.3 \times 10^{-17}$ & $1.4 \times 10^{-11}$ & $238.4 \pm 2.4$ \\
$\left(\mathrm{CH}_{3}\right)_{3} \mathrm{CI}$ & $6.8 \times 10^{-17}$ & $2.0 \times 10^{-11}$ & $230.1 \pm 2.8$
\end{tabular}

${ }^{a}$ Empirical estimate (see text). ${ }^{b}$ Based on the following $\Delta_{\mathrm{f}} H_{298}$ data, in $\mathrm{kJ} \mathrm{mol}^{-1}$ : from ref 9, (I) 106.8, $\left(\mathrm{CH}_{3}\right) 145.8 \pm 1.0,\left(\mathrm{CH}_{3} \mathrm{I}\right) 15.4 \pm$ $0.9,\left(\mathrm{C}_{2} \mathrm{H}_{5} \mathrm{I}\right)-9.0 \pm 0.9,\left(\mathrm{CH}_{3} \mathrm{CHICH}_{3}\right)-41.6 \pm 1.7$, and $\left(\left(\mathrm{CH}_{3}\right)_{3} \mathrm{CI}\right)$ $-72.0 \pm 2.2$; from ref. $25,\left(\mathrm{C}_{2} \mathrm{H}_{5}\right) 121.0 \pm 1.5,\left(\mathrm{CH}_{3} \mathrm{CHCH}_{3}\right) 90.0 \pm$ 1.7, and $\left(\left(\mathrm{CH}_{3}\right)_{3} \mathrm{C}\right) 51.3 \pm 1.8$.

An approximate idea of the rate constant for channel $1 \mathrm{~b}$ can be obtained by comparison with the analogous reaction

$$
\mathrm{H}+\mathrm{CH}_{4} \rightarrow \mathrm{H}_{2}+\mathrm{CH}_{3}
$$

for which $k_{10}$ is approximately $7.4 \times 10^{-19} \mathrm{~cm}^{3}$ molecule ${ }^{-1}$ $\mathrm{s}^{-1}$ at $298 \mathrm{~K}^{23}$ This is about $10^{-7}$ of $k_{1}$ at room temperature, and therefore it can be safely assumed that $\mathrm{H}$ abstraction plays a negligible role in reaction 1 . This is a reasonable comparison, bearing in mind the similar $\mathrm{C}-\mathrm{H}$ bond strengths in $\mathrm{CH}_{4}$ and $\mathrm{CH}_{3} \mathrm{I}$ of $440 \pm 1$ and $431 \pm 8 \mathrm{~kJ} \mathrm{~mol}^{-1}$, respectively. ${ }^{8}$ A similar assessment of $\mathrm{H}$-abstraction can be made for the heavier iodoalkanes. In these molecules all of the $\mathrm{C}-\mathrm{H}$ bonds are primary, and the rate constant for $\mathrm{H}$-abstraction from iodoalkanes was estimated as $n / 6$ times the rate constant for $\mathrm{H}+\mathrm{C}_{2} \mathrm{H}_{6}$ $\left(4.5 \times 10^{-17} \mathrm{~cm}^{3}\right.$ molecule $\mathrm{s}^{-1}$ at $\left.298 \mathrm{~K}^{23}\right)$, where $n$ is the number of $\mathrm{C}-\mathrm{H}$ bonds. The results are shown in Table 7 , and in all cases the $\mathrm{H}$-abstraction channel is minor. An earlier ab initio analysis of reaction 1 also showed that $\mathrm{H}$-atom abstraction is slow. ${ }^{3}$ This conclusion is reinforced by consideration of the measured kinetic isotope effect, $k_{1} / k_{1 \mathrm{~d}}$, which as seen from Figure 2 is slightly less than 1 . Had $\mathrm{C}-\mathrm{H}(\mathrm{D})$ bond breaking been the rate-determining step then the expected ratio $k_{1} / k_{1 \mathrm{~d}}$ at $298 \mathrm{~K}$ would have been around $8 .^{24}$ In summary, $k_{1}$ can be identified with $k_{1 \mathrm{a}}$.

$k_{X}$ at $298 \mathrm{~K}$ and the $\mathrm{C}-\mathrm{I}$ bond dissociation enthalpy $\mathrm{DH}_{298}$ are compared in Table $7 . \mathrm{DH}_{298}$ values were derived from literature enthalpies of formation for alkyl radicals ${ }^{25}$ and iodoalkanes, methyl radicals and I atoms. ${ }^{9}$ There is a monatonic trend for $k_{X}$ along the series of $\mathrm{CH}_{3} \mathrm{I}$ and primary to tertiary $\mathrm{C}-\mathrm{I}$ bonds. $\left(\mathrm{CH}_{3}\right)_{3} \mathrm{CI}$ has the weakest $\mathrm{C}-\mathrm{I}$ bond and is the most reactive alkyl iodide, but any correlation with $\mathrm{DH}_{298}$ is unclear. If the variation of $k_{1}$ to $k_{4}$ were due entirely to activation energy differences, a decrease of only $2.4 \mathrm{~kJ} \mathrm{~mol}^{-1}$ would account for the variation. This change is comparable to the experimental uncertainty in $\mathrm{DH}_{298}$. 


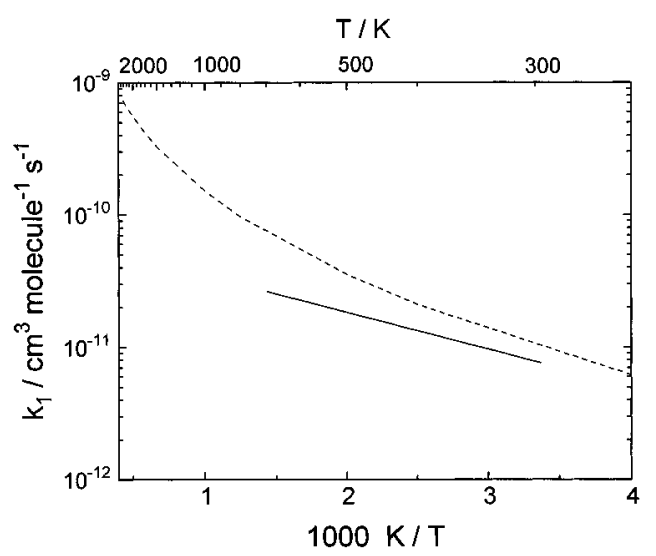

Figure 4. Comparison of measured $k_{1}$ for $\mathrm{H}+\mathrm{CH}_{3} \mathrm{I}$ (solid line, this work) with ab initio results (dashed line, ref 3 ).

Finally, we note that there is reasonable accord between the earlier $\mathrm{ab}$ initio prediction ${ }^{3}$ for $k_{1}$ and the present measurements: at $298 \mathrm{~K}$ the ab initio rate constant is higher than our experimental value by a factor of 1.15 , increasing to a factor of 2.9 at $760 \mathrm{~K}$. As seen in Figure 4, the discrepancies at higher temperatures arise from the higher curvature predicted for the Arrhenius plot of $k_{1}$. A possible explanation for this behavior is that variational effects are important in this system, and therefore that conventional transition state theory analysis overestimates $k_{1} \cdot{ }^{26}$ Such effects are generally more likely to be significant for reactions with smaller energy barriers, as is the case here.

\section{Conclusions}

The temperature dependences of the rate constants for reactions of $\mathrm{H}$ atoms with $\mathrm{CH}_{3} \mathrm{I}, \mathrm{CD}_{3} \mathrm{I}$, and $\mathrm{C}_{2} \mathrm{H}_{5} \mathrm{I}$ have been measured for the first time, and room temperature rate constants for $\mathrm{H}+\mathrm{CH}_{3} \mathrm{CHICH}_{3}$ and $\mathrm{H}+\left(\mathrm{CH}_{3}\right)_{3} \mathrm{CI}$ have also been determined. The results are consistent with I-abstraction as the main reaction pathway, and any contributions from I-substitution and $\mathrm{H}$-abstraction are small.

Acknowledgment. We thank the Robert A. Welch Foundation (Grant B-1174), the Air Force Office of Scientific Research, and the UNT Faculty Research Fund for financial support and the anonymous referees for valuable comments. Computer time was provided by the Wright Laboratory, Wright-Patterson AFB.

\section{References and Notes}

(1) Tsang, W., Miziolek, A. W., Eds. Halon Replacements: Technology and Science; ACS Symposium Series 611; Washington, DC, 1995.
(2) Noto, T.; Babushok, V.; Burgess, D. R., Jr.; Hamins, A.; Tsang, W.; Miziolek, A. 26th International Symposium on Combustion. 48.

(3) Marshall, P.; Misra, A.; Berry, R. J. Chem. Phys. Lett. 1997, 265,

(4) Leipunskii, I. O.; Morozov, I. I.; Tal'roze, V. L. Dokl. Phys. Chem. 1971, 198, 547. Russ. orig. p. 136.

(5) Levy, M. R.; Simons, J. P. J. Chem. Soc., Faraday Trans. 2 1975, 71,561 .

(6) Sillesen, A.; Ratajczak, E.; Pagsberg, P. Chem. Phys. Lett. 1993, $201,171$.

(7) Gilles, M. K.; Turnipseed, A. A.; Talukdar, R. K.; Rudich, Y.; Villalta, P. W.; Huey, L. G.; Burkholder, J. B.; Ravishankara, A. R. J. Phys. Chem. 1996, 100, 14005. 493.

(8) McMillen, D. F.; Golden, D. M. Ann. Rev. Phys. Chem. 1982, 33,

(9) Lias, S. G.; Bartmess, J. E.; Liebman, J. F.; Holmes, J. L.; Levin, R. D.; Mallard, W. G. Gas-Phase Ion and Neutral Thermochemistry; J. Phys. Chem. Ref. Data 1988, 17 (Suppl. 1).

(10) Schiesser, C. H.; Smart, B. A.; Tran, T.-A. Tetrahedron 1995, 51 , 3327.

(11) Curtiss, L. A.; Raghavachari, K.; Trucks, G. W.; Pople, J. A. J. Chem. Phys. 1991, 94, 7221.

(12) Glukhovtsev, M. N.; Pross, A.; McGrath, M. P.; Radom, L. J. Chem. Phys. 1995, 103, 1878

(13) Rebbert, R. E.; Lias, S. G.; Ausloos, P. Int. J. Chem. Kinet. 1973, $5,893$.

(14) Shi, Y.; Marshall, P. J. Phys. Chem. 1991, 95, 1654.

(15) Ding, L.; Marshall, P. J. Phys. Chem. 1992, 96, 2197.

(16) Goumri, A.; Yuan, W.-J.; Ding, L.; Shi, Y.; Marshall, P. Chem. Phys. 1993, 177, 233.

(17) Ding, L.; Marshall, P. J. Chem. Soc., Faraday Trans. 1993, 89, 419 .

(18) Baulch, D. L.; Duxbury, J.; Grant, S. J.; Montague, D. C. Evaluated Kinetic Data for High Temperature Reactions (Vol. 4); J. Phys. Chem. Ref. Data 1981, 10 (Suppl. 1).

(19) Lorenz, K.; Wagner, H. Gg.; Zellner, R. Ber. Bunsen-Ges. Phys. Chem. 1979, 83, 556.

(20) Umemoto, H.; Nakagawa, S.; Tsunashima, S.; Sato, S. J. Chem. Phys. 1988, 124, 259.

(21) Frisch, M. J.; Trucks, G. W.; Schlegel, H. B.; Gill, P. M. W.; Johnson, B. G.; Robb, M. A.; Cheeseman, J. R.; Keith, T.; Petersson, G. A.; Montgomery, J. A.; Raghavachari, K.; Al-Laham, M. A.; Zakrzewski, V. G.; Ortiz, J. V.; Foresman, J. B.; Peng, C. Y.; Ayala, P. Y.; Chen, W.; Wong, M. W.; Andres, J. L.; Replogle, E. S.; Gomperts, R.; Martin, R. L.; Fox, D. J.; Binkley, J. S.; Defrees, D. J.; Baker, J.; Stewart, J. P.; HeadGordon, M.; Gonzalez, C.; Pople, J. A. GAUSSIAN 94; Gaussian: Pittsburgh, PA, 1995.

(22) Gurvich, L., Veyts, I. V., Alcock, C. B., Eds. Thermodynamic Properties of Individual Substances; Hemisphere Pub. Corp.: New York, 1991.

(23) Baulch, D. L.; Cobos, C. J.; Cox, R. A.; Esser, C.; Frank, P.; Just, Th.; Kerr, J. A.; Pilling, M. J.; Troe, J.; Walker, R. W.; Warnatz, J. Evaluated Kinetic Data for Combustion Modeling; J. Phys. Chem. Ref. Data 1992, 21, 411 .

(24) Espenson, J. H. Chemical Kinetics and Reaction Mechanisms; McGraw-Hill: New York, 1981; p 203.

(25) Seakins, P. W.; Pilling, M. J.; Niiranen, J. T.; Gutman, D.; Krasnoperov, L. N. J. Phys. Chem. 1992, 96, 9847.

(26) Truhlar, D. G.; Isaacson, A. D.; Garrett, B. C. In Theory of Chemical Reaction Dynamics; Baer, M., Ed.; CRC Press: Boca Raton, FL, 1985; Vol. 4, Chapter 2 